Check for updates

Cite this: RSC Adv., 2017, 7, 32833

\title{
Syntheses, characterisation and solid-state study of alkali and ammonium BArF salts $\uparrow$
}

\author{
Lucas Carreras, (DD a Laura Rovira, ${ }^{a}$ Mónica Vaquero, (D) a Ignasi Mon, ${ }^{a}$ Eddy Martin, ${ }^{a}$ \\ Jordi Benet-Buchholz $z^{a}$ and Anton Vidal-Ferran (iD *ab
}

A new synthetic protocol for synthesising a number of BArF derivatives has been developed. Single crystal Xray analysis of an array of alkali metal and ammonium salts has allowed the determination of the coordination sphere and/or the map of short contacts of the positively charged atoms. The increasing number of coordination bonds and/or short contacts between the alkali metal cation and the surrounding atoms has been rationalised in terms of the size of the alkali metal centre. It has also been demonstrated that an increase in the number of coordination bonds and/or short contacts translates into longer $\mathrm{M}-\mathrm{F}$ distances. In the case of the ammonium BArF salts, the $\mathrm{N}-\mathrm{B}$ distances are shorter than the $\mathrm{M}-\mathrm{B}$ distances in the alkali metal BArF salts, indicating stronger interactions between the cationic nitrogen and the anionic boron than those between the boron and the alkali metal centres. Finally, a study of the structures of alkali metal hydrated and THF-solvated BArF salts showed that the interactions between the metal centre and the surrounding atoms depend not only on the size of the alkali metal centre but also on the occupancy of the first coordination sphere.

Received 26th May 2017

Accepted 21st June 2017

DOI: 10.1039/c7ra05928k

rsc.li/rsc-advances phenyl]borate anion $\left(\left[\mathrm{B}\left(3,5-\left(\mathrm{CF}_{3}\right)_{2} \mathrm{C}_{6} \mathrm{H}_{3}\right)_{4}\right]^{-} \text {, hereafter } \mathrm{BArF}\right)^{3 e}$ has gained significance within this group of fluorine-containing anions because of its stability and weak interaction with cations.

New and important applications for the BArF anion have been reported in recent years and have led to classical anions being replaced. ${ }^{3 \boldsymbol{b}-\boldsymbol{d}, \mathbf{5 - 1 3}}$ The BArF anion has been used in organic transformations as phase transfer catalyst, ${ }^{3 \boldsymbol{b}-\boldsymbol{d}}$ Lewis acid catalyst, ${ }^{5,6}$ in electrochemistry, ${ }^{7}$ in lithium batteries, ${ }^{8}$ as a component of ionic liquids ${ }^{9}$ and as a remover of lanthanide ions. ${ }^{\mathbf{1 0}}$ Regarding applications in enantioselective catalysis, the BArF anion has been widely used as a counterion in Ir-mediated hydrogenations, ${ }^{\mathbf{1 1}}$ and as regulation agent or additive for supramolecular catalysts. ${ }^{12,13}$

The synthesis of NaBArF was first reported by the Kobayashi group, who carried out the addition of arylmagnesium derivatives to boron reagents. ${ }^{3 b-e}$ Bergman and co-workers ${ }^{14}$ subsequently described more efficient synthetic protocols that avoided the use of magnesium metal as reagent. Analogous synthetic protocols have been developed for $\operatorname{LiBArF}^{3 \boldsymbol{h}, \mathbf{1 3 c}}$ and KBArF. ${ }^{3 h, 15}$ It has also been demonstrated that $\mathrm{RbBArF}^{\mathbf{1 2 d}}$ or $\mathrm{CsBArF}^{12 d}$ can be efficiently prepared by displacing the sodium cation in NaBArF with rubidium or cesium salts. ${ }^{16}$ The fact that RbBArF and CsBArF have lower solubility in water than NaBArF is the driving force behind the exchange of the cations bonded with the BArF moiety. Our group has pioneered the syntheses of a number of enantiopure ammonium BArF salts by displacement of the chloride with BArF anions in the corresponding amine hydrochlorides. ${ }^{12 b, d}$
${ }^{a}$ Institute of Chemical Research of Catalonia (ICIQ), The Barcelona Institute of Science and Technology, Av. Països Catalans 16, 43007 Tarragona, Spain.E-mail: avidal@ iciq.cat

${ }^{b}$ ICREA, Pg. Lluís Companys 23, 08010 Barcelona, Spain

$\dagger$ Electronic supplementary information (ESI) available: Experimental details, spectroscopic and crystallographic data. CCDC 1546291-1546302. For ESI and crystallographic data in CIF or other electronic format see DOI: 10.1039/c7ra05928k 


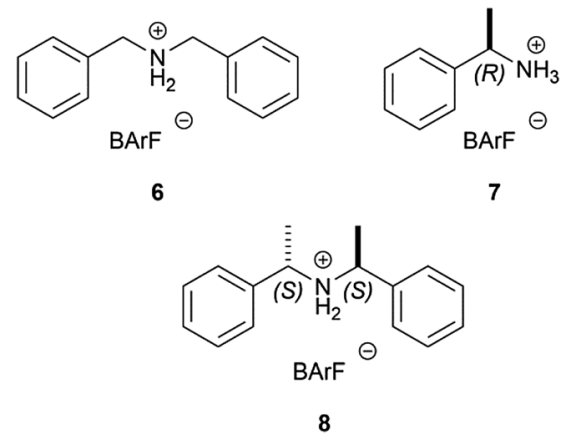

Fig. 1 Ammonium BArF derivatives.

Though alkali metal and some ammonium-based BArF salts were known in the literature, their structures in the solid-state had not been systematically determined and studied. ${ }^{17}$ Moreover, neither had systematic characterisation data of these compounds in solution ever been reported. ${ }^{18}$ Thus, we report herein our efforts to develop efficient methods for the preparation of a number of BArF salts and to characterise in the solidstate and in solution an array of alkali [i.e., LiBArF (1), NaBArF (2), KBArF (3), RbBArF (4) and CsBArF (5)] and ammonium BArF derivatives (structures 6-8, see Fig. 1). The present article provides valuable information on the spatial atomic arrangement, distances and geometries together with the coordination bonds and/or short contacts of the positively charged atoms in the solid-state.

\section{Experimental}

For the general information on the preparation methods for the BArF salts, see the ESI. $\uparrow$ The spectroscopic data of the BArF salts prepared using known methods can also be found in the ESI. $\dagger$

\section{Lithium tetrakis[3,5-bis(trifluoromethyl)phenyl]borate etherate, $\left(\mathrm{LiBArF} \cdot\left(\mathrm{EtO}_{2}\right)_{2}\right)$}

Lithium bis(trimethylsilyl)amide $(0.212 \mathrm{~g}, 1.23 \mathrm{mmol})$ was dissolved in $30 \mathrm{~mL}$ of diethyl ether. This solution was added dropwise over a solution of $\mathrm{HBArF} \cdot\left(\mathrm{EtO}_{2}\right)_{2}$ (ref. 19) $(1.24 \mathrm{~g}, 1.23$ $\mathrm{mmol}$ ) in $40 \mathrm{~mL}$ of diethyl ether at $-20{ }^{\circ} \mathrm{C}$. After stirring overnight at $-10{ }^{\circ} \mathrm{C}$, the solvent was concentrated under reduced pressure to dryness. The solid was dissolved in $6 \mathrm{~mL}$ of diethyl ether and the solution was cooled at $-78{ }^{\circ} \mathrm{C}$. Hexane $(20 \mathrm{~mL})$ was slowly added over this solution to precipitate the desired product. The solvent was filtered-off via cannula and dried under reduced pressure to afford the product $\mathrm{LiBArF} \cdot\left(\mathrm{Et}_{2} \mathrm{O}\right)_{2}$ as a white solid. Isolated $1.11 \mathrm{~g}, 89 \%$ yield. ${ }^{1} \mathrm{H} \mathrm{NMR}\left(\mathrm{CD}_{2} \mathrm{Cl}_{2}, 400\right.$ MHz): $\delta 7.72$ (br s, 8H), 7.57 (br s, 4H), 3.69 (q, $J=7.1 \mathrm{~Hz}, 8 \mathrm{H}$ ), $1.25(\mathrm{t}, J=7.1 \mathrm{~Hz}, 12 \mathrm{H}) \mathrm{ppm} .{ }^{13} \mathrm{C}\left\{{ }^{1} \mathrm{H}\right\} \mathrm{NMR}\left(\mathrm{CD}_{2} \mathrm{Cl}_{2}, 126 \mathrm{MHz}\right)$ : $\delta 162.2\left(\mathrm{q}, J_{\mathrm{C}-\mathrm{B}}=50 \mathrm{~Hz}\right), 135.2,129.3\left(\mathrm{qm}, J_{\mathrm{C}-\mathrm{F}}=32 \mathrm{~Hz}\right), 125.0$ $\left(\mathrm{q}, J_{\mathrm{C}-\mathrm{F}}=272 \mathrm{~Hz}\right), 117.9(\mathrm{~m}), 67.0,14.7 \mathrm{ppm} .{ }^{19} \mathrm{~F}\left\{{ }^{1} \mathrm{H}\right\}$ NMR $\left(\mathrm{C}_{4} \mathrm{D}_{8} \mathrm{O}, 376 \mathrm{MHz}\right): \delta-63.2(24 \mathrm{~F}) \mathrm{ppm} .{ }^{11} \mathrm{~B}\left\{{ }^{1} \mathrm{H}\right\} \mathrm{NMR}\left(\mathrm{C}_{4} \mathrm{D}_{8} \mathrm{O}\right.$, $128 \mathrm{MHz}): \delta-8.6 \mathrm{ppm} .{ }^{7} \mathrm{Li} \mathrm{NMR}\left(\mathrm{D}_{2} \mathrm{O}, 194 \mathrm{MHz}\right): \delta-0.4 \mathrm{ppm}$. IR (neat, $\mathrm{cm}^{-1}$ ): $1624,1611,1354,1274,1111,1101 . \mathrm{Mp}=145-$ $148{ }^{\circ} \mathrm{C}$. Content in Li by ICP-AES: calcd $0.68 \%$, found $0.67 \%$. IR and ${ }^{1} \mathrm{H}$ NMR data were in agreement with those previously reported for LiBArF $4 \mathrm{H}_{2} \mathrm{O}{ }^{3 h}$

\section{Potassium tetrakis[3,5-bis(trifluoromethyl)phenyl]borate, (KBArF)}

Potassium bis(trimethylsilyl)amide $(0.18 \mathrm{~g}, 0.85 \mathrm{mmol})$ was dissolved in $50 \mathrm{~mL}$ of diethyl ether. This solution was added dropwise over a solution of $\mathrm{HBArF} \cdot\left(\mathrm{EtO}_{2}\right)_{2}(0.86 \mathrm{~g}, 0.85 \mathrm{mmol})$ in $30 \mathrm{~mL}$ of diethyl ether at $-20{ }^{\circ} \mathrm{C}$. After stirring overnight at $-20{ }^{\circ} \mathrm{C}$, the solvent was concentrated under reduced pressure to dryness. The solid was dissolved in $6 \mathrm{~mL}$ of diethyl ether and the solution was cooled at $-78{ }^{\circ} \mathrm{C}$. Hexane $(20 \mathrm{~mL})$ was slowly added over this solution to precipitate the desired product. The solvent was filtered-off via cannula and dried under reduced pressure to afford the product KBArF as a white solid. Isolated $0.61 \mathrm{~g}, 80 \%$ yield. ${ }^{1} \mathrm{H}$ NMR $\left(\mathrm{C}_{4} \mathrm{D}_{8} \mathrm{O}, 400 \mathrm{MHz}\right): \delta 7.79$ (br s, 8H), 7.58 (br s, $4 \mathrm{H})$ ppm. ${ }^{13} \mathrm{C}\left\{{ }^{1} \mathrm{H}\right\} \operatorname{NMR}\left(\mathrm{C}_{4} \mathrm{D}_{8} \mathrm{O}, 126 \mathrm{MHz}\right): \delta 162.8\left(\mathrm{q}, J_{\mathrm{C}-\mathrm{B}}=50\right.$ $\mathrm{Hz}), 135.6,130.0\left(\mathrm{qm}, J_{\mathrm{C}-\mathrm{F}}=32 \mathrm{~Hz}\right), 125.5$ (q, $\left.J_{\mathrm{C}-\mathrm{F}}=272 \mathrm{~Hz}\right)$, $118.2(\mathrm{~m})$ ppm. ${ }^{19} \mathrm{~F}\left\{{ }^{1} \mathrm{H}\right\}$ NMR $\left(\mathrm{C}_{4} \mathrm{D}_{8} \mathrm{O}, 376 \mathrm{MHz}\right): \delta-63.2$ (24F) ppm. ${ }^{11} \mathrm{~B}\left\{{ }^{1} \mathrm{H}\right\}$ NMR $\left(\mathrm{C}_{4} \mathrm{D}_{8} \mathrm{O}, 128 \mathrm{MHz}\right): \delta-8.6 \mathrm{ppm}$. IR (neat, $\mathrm{cm}^{-1}$ ): $1612,1357,1281,1187,1124 . \mathrm{Mp} \geq 350{ }^{\circ} \mathrm{C}^{20}$ Content in K by ICP-OES: calcd $4.33 \%$, found $4.40 \%$. IR and ${ }^{1} \mathrm{H}$ NMR data were in agreement with those previously reported. ${ }^{\mathbf{1 5}}$

\section{(R)-(1-Phenylethyl)ammonium tetrakis[3,5-} bis(trifluoromethyl)-phenyl]borate, (7)

NaBArF (0.28 g, $0.32 \mathrm{mmol})$ was mixed with (R)-(1-phenylethyl) ammonium hydrochloride ${ }^{21}(0.05 \mathrm{~g}, 0.32 \mathrm{mmol})$ in $5 \mathrm{~mL}$ of $\mathrm{Et}_{2} \mathrm{O}$ and allowed to react overnight. Filtration and evaporation of the solvent yielded the target compound as a white solid $(0.30 \mathrm{~g}$, 95\% yield). ${ }^{1} \mathrm{H}$ NMR $\left(\mathrm{CD}_{2} \mathrm{Cl}_{2}, 400 \mathrm{MHz}\right): \delta 7.73$ (br s, 8H), 7.58$7.52(\mathrm{~m}, 7 \mathrm{H}), 7.44-7.39(\mathrm{~m}, 2 \mathrm{H}), 5.91$ (br s, 3H), $4.90(\mathrm{q}, J=$ $6.9 \mathrm{~Hz}, 1 \mathrm{H}), 1.91(\mathrm{~d}, J=6.9 \mathrm{~Hz}, 3 \mathrm{H}) \mathrm{ppm} .{ }^{13} \mathrm{C}\left\{{ }^{1} \mathrm{H}\right\} \mathrm{NMR}\left(\mathrm{CD}_{2} \mathrm{Cl}_{2}\right.$, $100 \mathrm{MHz}$ ): $\delta 162.2\left(\mathrm{q}, J_{\mathrm{C}-\mathrm{B}}=49 \mathrm{~Hz}\right), 135.2,133.7,132.1,130.9$, $129.3\left(\mathrm{qm}, J_{\mathrm{C}-\mathrm{F}}=31 \mathrm{~Hz}\right), 126.7,125.1\left(\mathrm{q}, J_{\mathrm{C}-\mathrm{F}}=272 \mathrm{~Hz}\right), 117.9$ (m), 56.9, 20.1 ppm. ${ }^{19} \mathrm{~F}\left\{{ }^{1} \mathrm{H}\right\}$ NMR $\left(\mathrm{CD}_{2} \mathrm{Cl}_{2}, 376 \mathrm{MHz}\right): \delta-63.3$ (24F) ppm. ${ }^{11} \mathrm{~B}\left\{{ }^{1} \mathrm{H}\right\}$ NMR $\left(\mathrm{CD}_{2} \mathrm{Cl}_{2}, 128 \mathrm{MHz}\right): \delta-8.6 \mathrm{ppm}$. IR (neat, $\mathrm{cm}^{-1}$ ): 3683, 3606, 3323, 3281, 2857, 1612, 1492, 1353, 1275, 1098. $\mathrm{Mp}=149-154{ }^{\circ} \mathrm{C} .[\alpha]_{\mathrm{D}}^{25}+1.0\left(c 1.03, \mathrm{CH}_{3} \mathrm{OH}\right) . \mathrm{MS}$ (ESI): $m / z$ calcd for $\mathrm{C}_{8} \mathrm{H}_{12} \mathrm{~N}$ : 122.1; found: $122.1[\mathrm{M}-\mathrm{BArF}]^{+}$. Elemental analysis calcd (\%) for $\mathrm{C}_{41} \mathrm{H}_{24} \mathrm{BF}_{24} \mathrm{~N}$ : C 48.75, H 2.45, N 1.42; found: C 48.12, H 2.49, N 1.49 .

Details on the determination of single crystal X-ray diffraction structures and general structural comments were included in the ESI $\dagger$ of this article.

\section{Results and discussion}

Syntheses of alkali metal and ammonium BArF derivatives

Though the preparation of LiBArF and KBArF was known in the literature, ${ }^{3 \boldsymbol{h}, \mathbf{1 3 c}, \mathbf{1 5}}$ these syntheses require the use of organomagnesium intermediates and aqueous work-ups. Thus, we envisaged that these compounds could alternatively be prepared via an acid-base reaction (Scheme 1) between the corresponding acid of BArF (9) and the corresponding alkali 


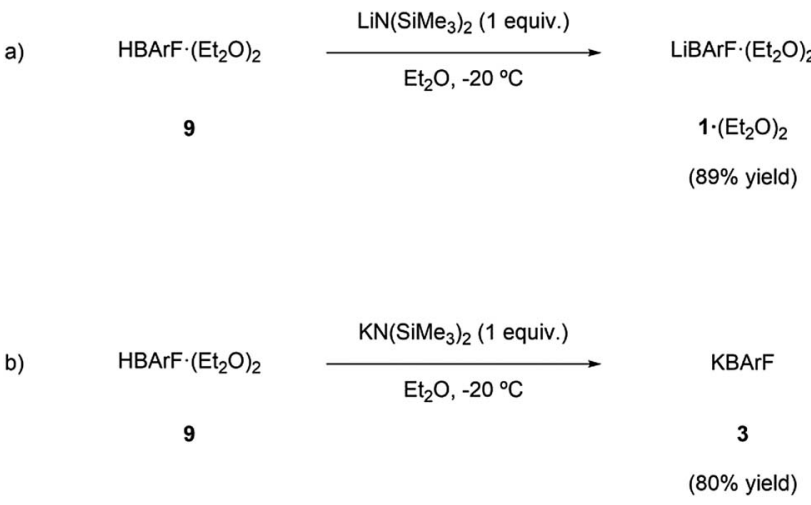

Scheme 1 Syntheses of (a) $\operatorname{LiBArF} \cdot\left(\mathrm{Et}_{2} \mathrm{O}\right)_{2}$ and (b) KBArF.

metal bis(trimethylsilyl)amide (i.e., $\operatorname{LiN}\left(\mathrm{SiMe}_{3}\right)_{2}$ or $\mathrm{KN}\left(\mathrm{SiMe}_{3}\right)_{2}$, respectively) in a low boiling point organic solvent.

The low boiling point of the liquid components of the mixture would facilitate the isolation of the desired compound by evaporation in vacuo. This synthetic procedure was followed and $\mathrm{LiBArF} \cdot\left(\mathrm{Et}_{2} \mathrm{O}\right)_{2}$ and $\mathrm{KBArF}$ were isolated in high yield. It should be noted that this preparation method does not require separating the target compound from other salts.

The preparation of RbBArF, CsBArF and the ammonium BArF derivatives is well documented and relies on a counteranion exchange reaction around the alkali metal or ammonium groups. ${ }^{12 d}$ The difference in the solubility of the target BArF derivative and the by-product of the reaction (i.e. the salt arising from the combination of the cation initially bound to the BArFcontaining reagent and the anion initially bound to the alkali metal or ammonium groups) constitutes the driving force of the reaction and shifts the equilibrium. The preparation methods for the required starting materials and BArF salts have been detailed in the ESI. $\dagger$

\section{Characterisation in solution of alkali metal and ammonium BArF derivatives}

The whole array of BArF salts was characterised using standard spectroscopic techniques (IR and ${ }^{1} \mathrm{H},{ }^{7} \mathrm{Li},{ }^{11} \mathrm{~B},{ }^{13} \mathrm{C},{ }^{19} \mathrm{~F}$ and ${ }^{133} \mathrm{Cs}$ NMR spectroscopy). ${ }^{19} \mathrm{~F}\left\{{ }^{1} \mathrm{H}\right\}$ and ${ }^{11} \mathrm{~B}\left\{{ }^{1} \mathrm{H}\right\}$ NMR spectra showed

Table $1{ }^{11} \mathrm{~B}\left\{{ }^{1} \mathrm{H}\right\}$ and ${ }^{19} \mathrm{~F}\left\{{ }^{1} \mathrm{H}\right\}$ NMR chemical shifts (ppm) for the alkali metal (in $\mathrm{C}_{4} \mathrm{D}_{8} \mathrm{O}$ ) and ammonium (in $\mathrm{CD}_{2} \mathrm{Cl}_{2}$ ) BArF salts

\begin{tabular}{llll}
\hline Entry & Compound & ${ }^{11} \mathrm{~B}\left\{{ }^{1} \mathrm{H}\right\}^{a}$ & ${ }^{19} \mathrm{~F}\left\{{ }^{1} \mathrm{H}\right\}^{b}$ \\
\hline 1 & $\mathrm{LiBArF} \cdot\left(\mathrm{Et}_{2} \mathrm{O}\right)_{2}$ & -8.6 & -63.2 \\
2 & $\mathrm{NaBArF}$ & -8.6 & -63.1 \\
3 & $\mathrm{KBArF}$ & -8.6 & -63.2 \\
4 & $\mathrm{RbBArF}$ & -8.5 & -63.3 \\
5 & $\mathrm{CsBArF}$ & -8.6 & -63.1 \\
6 & $\mathbf{6}$ & -8.5 & -63.0 \\
7 & 7 & -8.5 & -63.3 \\
8 & $\mathbf{8}$ & -8.6 & -63.3
\end{tabular}

${ }^{a}{ }^{11} \mathrm{~B}\left\{{ }^{1} \mathrm{H}\right\}$ chemical shifts are quoted in ppm relative to trimethyl borate (TMB) in $\mathrm{C}_{4} \mathrm{D}_{8} \mathrm{O} .{ }^{b}{ }^{19} \mathrm{~F}\left\{{ }^{1} \mathrm{H}\right\}$ chemical shifts are quoted in ppm relative to trifluoroacetic acid (TFA) in $\mathrm{C}_{4} \mathrm{D}_{8} \mathrm{O}$. only a singlet for the signals of the BArF anion with very small differences in the chemical shifts (see Table 1 ). ${ }^{1} \mathrm{H}$ was in agreement with the proposed structures and ${ }^{13} \mathrm{C}\left\{{ }^{1} \mathrm{H}\right\}$ NMR showed the expected set of signals for the four equivalent 3,5bis(trifluoromethyl)phenyl groups and the corresponding organic moieties in the case of the ammonium BArF salts. IR spectroscopy also confirmed the structure of the BArF salts with intense bands in the $\mathrm{C}=\mathrm{C}$ stretching $\left(c a .1600-1650 \mathrm{~cm}^{-1}\right)$ and C-F stretching ( $c a .1100-1350 \mathrm{~cm}^{-1}$ ) vibration regions. Intense bands in the $\mathrm{N}^{+}-\mathrm{H}$ stretching region (ca. $2700-3000 \mathrm{~cm}^{-1}$, broad) were also observed for the ammonium BArF salts.

\section{Crystal structure description and analysis}

One of the key structural features of the BArF anion is that its negative charge is distributed over its skeleton, in which the electronegative fluorine atoms play a major role in accommodating the single negative charge of the boron.

The distribution of this single negative charge over a multitude of atoms renders the BArF anion both weakly nucleophilic and basic, which poses questions about the coordination sphere (CS) of the cationic species and about the number of weak interactions (or short contacts) required for the single positive charge of the alkali metal or ammonium cation to be compensated. Regarding the alkali metal BArF salts, we reasoned that the different charge densities in the alkali metal cationic series (i.e., $\mathrm{Li}^{+}, \mathrm{Na}^{+}, \mathrm{K}^{+}, \mathrm{Rb}^{+}$and $\mathrm{Cs}^{+}$) arising from the differences in the cationic radii would translate into a different number of interactions between the fluorine atoms or solvate molecules and the cation in each alkali metal BArF derivative. In terms of the ammonium BArF derivatives, we reasoned that the size and topology of the backbones of the substituents at nitrogen would affect the number of interactions between the electronegative atoms present in the BArF anion or solvate molecules and the nitrogen cation. We considered that determining the coordination spheres, the map of short contacts and the space-charge compensation map for the positively charged atom in a number of alkali metal BArF and ammonium BArF derivatives would be highly useful for understanding the role of these interesting derivatives in catalytic processes, ${ }^{3 b-d, 5,6,11-13}$ electrochemistry ${ }^{7,8}$ and other applications. ${ }^{9,10}$ Thus, we turned our attention to obtaining single crystals suitable for X-ray analysis for the whole series of BArF derivatives and to performing the corresponding structural studies by X-ray analysis. Single crystals suitable for X-ray analysis were obtained for the whole series of alkali metal BArF salts and the ammonium derivatives studied. Molecules of solvate were incorporated into the crystal structure in some structures (i.e., LiBArF-4THF, NaBArF-6THF, CsBArF-7THF and CsBArF-DCM). Moreover, residual water molecules from the solvent were incorporated into the crystal structure in some cases (i.e., LiBArF, NaBArF, KBArF, RbBArF and 7).

Alkali metal BArF salts. The detailed single crystal X-ray diffraction study not only confirmed the structure of the compounds but, as intended, also provided important information on how the BArF moiety and solvent molecules interact with the alkali metal (selected distance values in the solid-state 
are summarised in the discussion that follows and in Table 2). The asymmetric unit in the structure of LiBArF contains a quarter of one BArF molecule (corresponding to $S_{4}$ symmetry), a quarter of one lithium atom and 0.68 highly disordered molecules of water. The water molecules crystallised in two disordered ways forming two types of structures. In Fig. 2A, an octahedral geometry can be observed which is formed by two water oxygen atoms ( $\mathrm{O} 1$ and $\left.\mathrm{O}_{2}^{\prime}\right)$, and four fluorine atoms from neighbouring $\mathrm{CF}_{3}$ groups (F4, F4A, F4B and F4C). In Fig. 2B, a trigonal bipyramidal geometry can be observed. In this case three water oxygen atoms $(\mathrm{O} 1, \mathrm{O} 2$ and $\mathrm{O} 2 \mathrm{~A})$ are coordinated in a central plane to the lithium atom and two fluorine atoms (F4 and F4A) are placed in the axial positions of the trigonal bipyramid. The octahedral geometry is minor (28\%), while the trigonal bipyramidal geometry is major (72\%). The BArF cation is disordered in two orientations and shows a slightly distorted tetrahedral geometry. Interestingly, a higher solvation around the Li cation translates into a longer Li-F distance (i.e., 2.287(3) $\AA$ in structures LiBArF-A or LiBArF-B with two or three solvation molecules of water, respectively, and $3.34 \AA$ in structure LiBArF $\left.\cdot 4 \mathrm{H}_{2} \mathrm{O}\right){ }^{22}$ This observation indicates that an increased water solvation leads to swarming of the ion coordination sphere and translates into a separation of the anion and the cation. The single crystal structure obtained for NaBArF is isostructural to that obtained for LiBArF. An analysis of the structure collected at room temperature has already been reported. ${ }^{23}$ However, our discussion uses the data collected by us at $100 \mathrm{~K}$ to aid comparison. The asymmetric unit of NaBArF contains a quarter of one BArF molecule (corresponding to $S_{4}$ symmetry), a quarter of one sodium atom and 0.59 highly disordered molecules of water. As in the case of LiBArF, NaBArF adopts an octahedral geometry (Fig. 2C), where the two oxygen atoms from the water molecules ( $\mathrm{O} 1$ and $\mathrm{O} 2{ }^{\prime}$ ) and the four fluorine atoms (F4-F4C) interact with the sodium atom, and a trigonal bipyramidal geometry (Fig. 2D), where the sodium atom interacts with two fluorine atoms (F4 and F4A) and with the three oxygen atoms from the water molecules $(\mathrm{O} 1, \mathrm{O} 2$ and $\mathrm{O} 2 \mathrm{~A})$. In contrast to LiBArF, the octahedral geometry is major $(62 \%)$, while the trigonal bipyramidal geometry is minor $(38 \%)$. The BArF cation is disordered in two orientations and shows a slightly distorted tetrahedral geometry. The crystal structure obtained for KBArF (see Fig. 2E) corresponds to the space group
$P 4 / n$ but is not isostructural to the structure of lithium and sodium BArF salts. In the asymmetric unit we identified a quarter of one BArF molecule (corresponding to $S_{4}$ symmetry), a quarter of a potassium atom and $1 / 8$ of a water molecule. The BArF anion displayed as expected a slightly distorted tetrahedral geometry. The potassium atom is surrounded by eight fluorine atoms (F3, F4 and their symmetry equivalents) and one oxygen atom (O1W) from a water molecule (see Fig. 2E). The shortest $\mathrm{K}-\mathrm{F}$ distances in KBArF are $2.61 \AA$ (F3') and $2.73 \AA$ (F4') and the $\mathrm{K}-\mathrm{O}$ distance is $2.81 \AA$ (O1W, see Table 2$)$. RbBArF crystals were isostructural to those obtained for KBArF (compare Fig. 2E with F). The asymmetric unit contains a quarter of one BArF molecule, a quarter of one rubidium atom and a quarter of one water molecule. The BArF anion is located on a rotation-reflection axis, presents $S_{4}$ symmetry and displays a slightly distorted tetrahedral geometry. The shortest $\mathrm{Rb}-\mathrm{F}$ distances are $2.99 \AA$ (F3) and $2.93 \AA$ (F4), and the $\mathrm{Rb}-\mathrm{O}$ distance is $2.94 \AA$ (O1W, see Table 2). Finally, the crystal structure of CsBArF (see Fig. 2G) was studied. The asymmetric unit of this salt contains a quarter of a BArF molecule showing a $S_{4}$ symmetry, a quarter of a cesium atom located on a four-fold rotation axis and a quarter of a $\mathrm{CH}_{2} \mathrm{Cl}_{2}$ molecule. The BArF cation is disordered in two orientations and presents a slightly distorted tetrahedral geometry. In this case, the crystal symmetry changes from the $P 4 / n$ space group observed in the other BArF salts to the $P 4 / n c c$ space group. Since the cesium atom is larger than potassium and rubidium, a higher number of interactions with the surrounding atoms were observed. Twelve interactions with the fluorine atoms F3, F4 and F5 (and their symmetry equivalents) and two with the chlorine atoms from the crystallisation solvent with distances ranging from 3.04 to $3.81 \AA$ (see Fig. $2 \mathrm{G}$ and Table 2) were observed. It is interesting to note the high number of interactions observed for the cesium derivative (fourteen), which is one of the highest reported in the literature for a cesium centre. ${ }^{24}$

Ammonium BArF salts. Single crystals suitable for X-ray analysis were obtained for compounds 6-8 and crystallographic studies confirmed the structures of the compounds (see Fig. 3). Moreover, interesting information was obtained regarding the short contact interactions between some atoms in the BArF moieties and the cationic nitrogen atoms. Relevant information is listed in the discussion that follows and in

Table 2 Selected distances $(\AA)$ for alkali metal BArF salts ${ }^{a}$

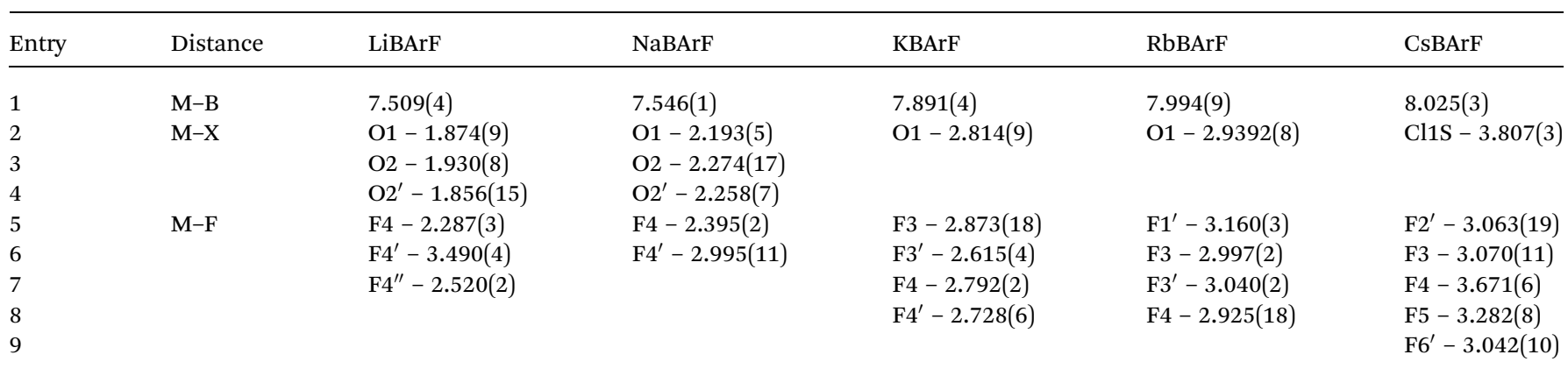

${ }^{a}(')$ the prime code corresponds to disordered atoms. 


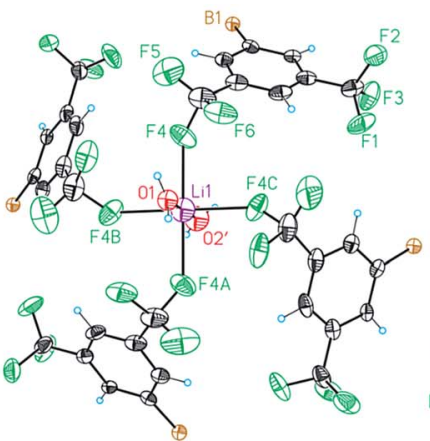

A (1, LiBArF)

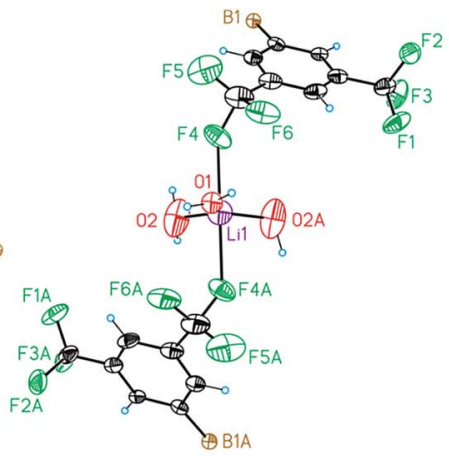

$\mathrm{B}(1, \mathrm{LiBArF})$

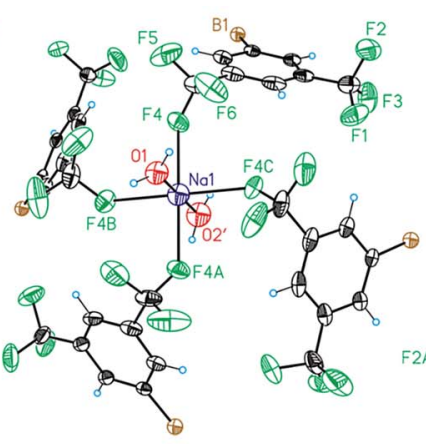

C (2, NaBArF)

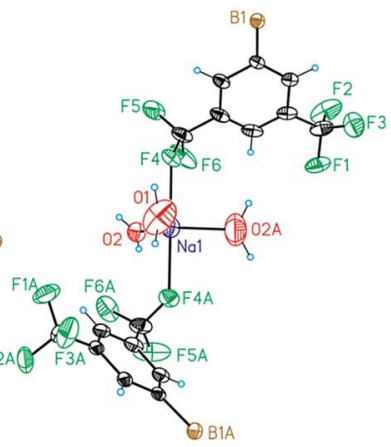

D (2, NaBArF)

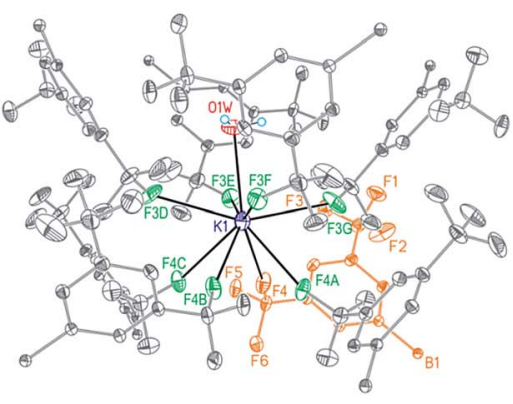

$E(3, \mathrm{KBArF})$

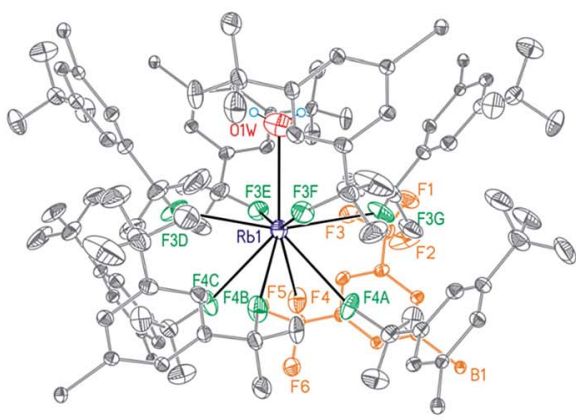

$F(4, \operatorname{RbBArF})$

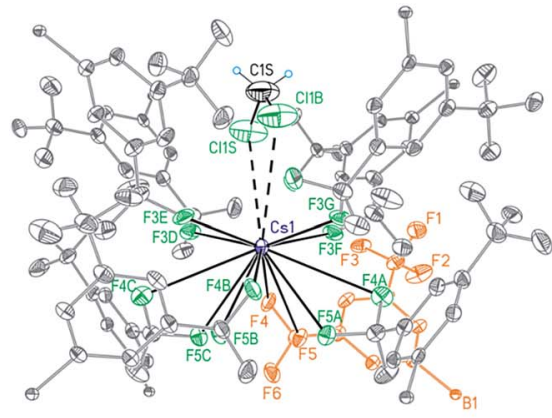

$\mathrm{G}(5, \mathrm{CsBArF})$

Fig. 2 Crystal structure of the alkali metal BArF derivatives. Some phenyl rings from the BArF unit have been omitted for the sake of clarity. Colour scheme: C: black, H: blue, B: orange, F: green, Li: violet, O: red; $\mathrm{Na} / \mathrm{K} / \mathrm{Rb} / \mathrm{Cs}$ : purple. Atomic displacement ellipsoids are drawn at a $50 \%$ probability level. For LiBArF: octahedral (A) and trigonal bipyramidal (B) geometries, in which fluorine atoms F4A, F4B and F4C are symmetry equivalent to F4. For NaBArF: octahedral (C) and trigonal bipyramidal (D) geometries, in which fluorine atoms F4, F4A, F4B and F4C are symmetry equivalent. For KBArF: see structure (E) for the geometry, in which fluorine atoms F3D, F3E, F3F and F3G are symmetry equivalent to F3 and F4A, $F 4 B$ and F4C to F4. For RbBArF: see structure (F) for the geometry, in which fluorine atoms F3D, F3E, F3F and F3G are symmetry equivalent to F3 and F4A, F4B and F4C to F4. For CsBArF: see structure (G) for the geometry, in which fluorine atoms F3D, F3E, F3F, F3G, F4A, F4B, F4C, F5A, F5B and $\mathrm{F} 5 \mathrm{C}$ are symmetry equivalent to F3, F4 and F5, respectively.

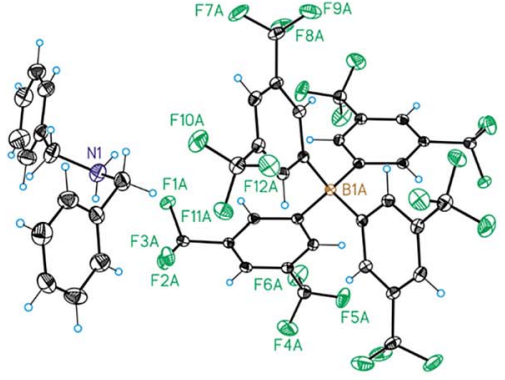

$A(6)$

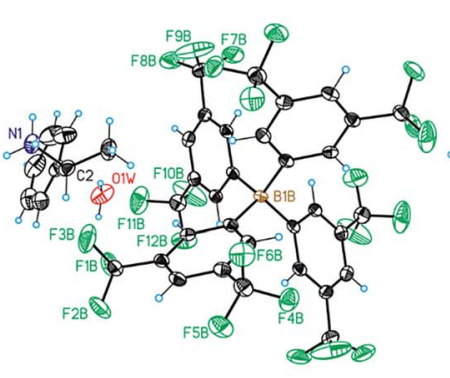

$B(7)$

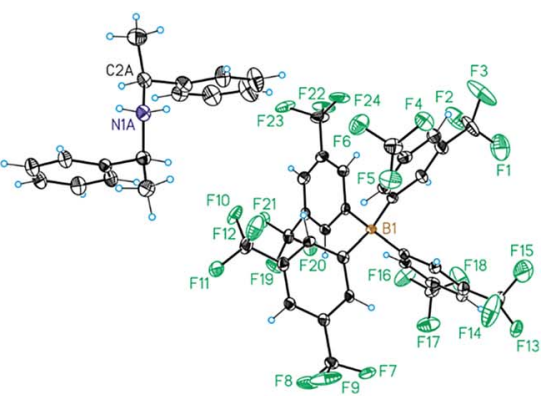

C (8)

Fig. 3 Crystal structure of the ammonium BArF derivatives 6-8 ((A) structure of 6; (B) structure of 7; (C) structure of 8). Colour scheme: C: black, $\mathrm{H}$ : blue, B: orange, F: green, N: purple, O: red. Atomic displacement ellipsoids are drawn at a 50\% probability level.

Table 3. The asymmetric unit in the structure of 6 contains two halves of the BArF anion and the dibenzylammonium counterion, with a slightly distorted $T_{\mathrm{d}}$ symmetry (see Fig. $3 \mathrm{~A}$ ). The two independent BArF cations have a slightly distorted tetrahedral geometry and show only $C_{2}$ symmetry because the two aromatic rings present in the asymmetric unit display different orientations. Short contacts were observed between the hydrogen atoms from the nitrogen and the fluorine atoms. Additional short contacts were observed for the hydrogen atoms from the ammonium benzyl aromatic rings and fluorine atoms. (R)-(1-Phenylethyl)ammonium BArF (7) crystallised in the space group $C 2$ with one ammonium cation, two half BArF anions and one water molecule in the asymmetric unit. The two independent BArF structures have a slightly distorted tetrahedral 
Table 3 Selected shortest distances $(\AA)$ for ammonium BArF salts 6-8

\begin{tabular}{lllll}
\hline Entry & Distance & $\mathbf{6}$ & 7 & $\mathbf{8}$ \\
\hline 1 & $\mathrm{~N}-\mathrm{B}$ & $6.724(2)$ & $7.477(3)$ & $6.778(6)$ \\
2 & $\mathrm{~N}-\mathrm{F}$ & $3.058(2)$ & $2.959(3)$ & $2.995(5)$ \\
3 & $\mathrm{~N}-\mathrm{O}$ & - & $2.705(4)$ & - \\
\hline
\end{tabular}

geometry and show only $C_{2}$ symmetry because the two aromatic rings in the asymmetric unit present different orientations. Fig. 3B shows the structure obtained using only one half of the expanded BArF anions. A short contact was observed between the asymmetric hydrogen atom from the counterion and a fluorine atom from the BArF group. Additional short contacts were observed between the water molecule and a fluorine atom, and between the water molecule and the phenyl moiety from the cation. Remarkably, the water molecule forms a hydrogen bond with the cationic nitrogen atom (N1-O1W $=2.71 \AA$ A). $(S, S)$-Bis-(1phenylethyl)ammonium BArF (8) crystallised in the triclinic space group $P 1$. The asymmetric unit in the structure contains, in this case, a whole BArF molecule which has $C_{1}$ symmetry with a slightly distorted tetrahedral geometry for the carbon atom and the secondary ammonium counterion which has a slightly distorted molecular $T_{\mathrm{d}}$ symmetry. The solid-state structure of the salt is displayed in Fig. 3C. In this case, no remarkable short contacts were observed. The steric hindrance of the methyl substituents in the ammonium could account for the lack of interactions with the BArF moiety.

Tetrahydrofuran-solvated alkali metal BArF salts. Having determined the distances and geometries within the crystal structures of alkali metal BArF salts, we turned our attention to performing a similar study on a set of solvates from the same derivatives. We anticipated that the geometries and map of short contacts between the BArF anion and the solvated alkali metal cations would be different from those obtained for the alkali metal BArF structures. The oxophilicity of alkali metal cations ${ }^{25}$ led us to reason that crystallisation of the BArF salts in an oxygen-containing solvent such as tetrahydrofuran (THF) would lead to the corresponding solvates. Crystals suitable for X-ray analysis were obtained following this strategy. The most relevant features of the crystal structures studied are described in the discussion that follows. THF-solvated LiBArF crystallised with four molecules of solvent around the $\mathrm{Li}$ atom in a tetrahedral manner (see Fig. 4A). ${ }^{26}$ THF-solvates of NaBArF and $\mathrm{CsBArF}^{27}$ could also be successfully crystallised. In the case of the NaBArF THF-solvate, six molecules of THF in a slightly distorted octahedral geometry around the Na were observed, whereas for CsBArF, seven molecules of THF were displayed around the Cs centre in a monocapped trigonal prismatic geometry. As expected, the coordination number of the metal centres increased as did the size of the metal. Not surprisingly, the M-B distances for the THF-solvates became longer as the size of the cation increased ( $7.94 \AA$ for LiBArF $4 \mathrm{THF}, 8.74 \AA$ for NaBArF - 6THF and $9.09 \AA$ for CsBArF - 7THF). As a direct effect of the longer $\mathrm{M}-\mathrm{B}$ distances for the THF-solvates compared with the alkali metal derivatives, no remarkable short contacts were observed between the fluorine atoms and the corresponding alkali metal cation in any of the three structures studied.

Discussion on solid-state structures of BArF salts. The detailed single crystal X-ray diffraction study that was performed provided important information about their structure. A few trends were observed regarding the distances between the boron atom of the BArF motif and the cationic alkali metal or ammonium centres. For the series of alkali metal BArF salts (Table 2), the M-B distance became longer as the ionic radius of the cation increased. In terms of the ammonium BArF salts (compounds 6-8), it is interesting to point out that the ammonium-based structures had an $\mathrm{N}-\mathrm{B}$ distance ranging from 6.72 to $7.48 \AA$ (see Table 3), which is shorter than the B-Li distance (7.51 $\AA$; see Table 2), despite the fact that the lithium had a smaller cationic radius than that of $\mathrm{NH}_{4}{ }^{+}$(radii for $\mathrm{Li}^{+}$and $\mathrm{NH}_{4}{ }^{+}$are described as $0.79 \AA$ and $2.11 \AA$, respectively). ${ }^{28}$ Moreover, a trend between the steric bulkiness around the nitrogen atom and the N-B distances could not be established: the N-B distance of the primary ammonium BArF derivative 7 was higher than those of the secondary ammonium BArF derivatives 6 and 8 (compare distances in entry 1 of Table 3). Other relevant structural parameters in the alkali metal BArF salts are the number of coordination bonds and/or short contacts identified in the crystal structure. A representation of the number of these two types of interaction compared with the ionic radius of the

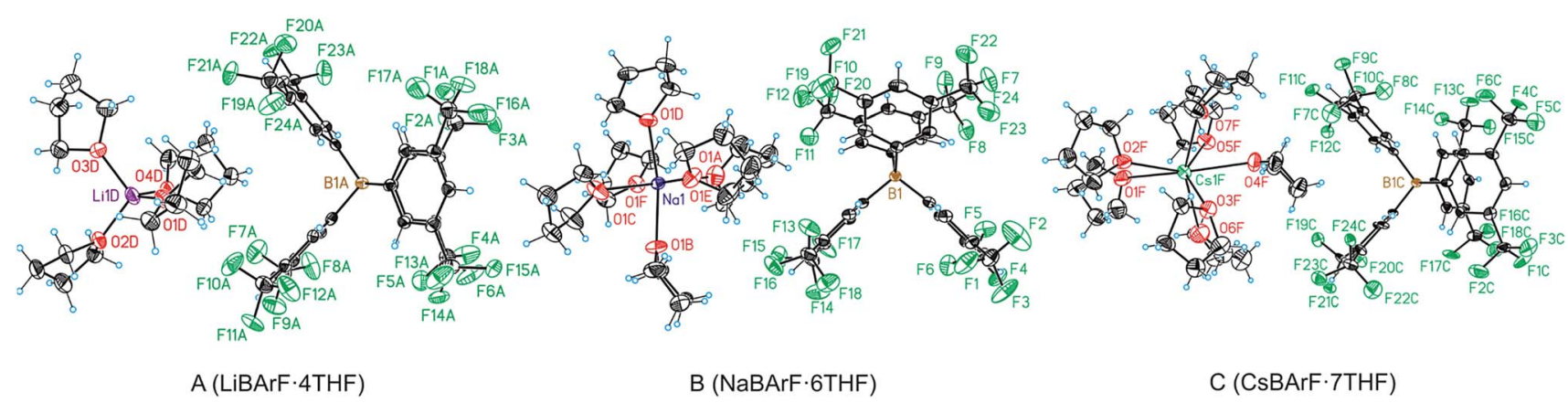

Fig. 4 Crystal structure of alkali metal BArF solvates ((A) structure of LiBArF.4THF; (B) structure of NaBArF.6THF; (C) structure of CsBArF.7THF). Colour scheme: C: black, H: blue, B: orange, F: green, Li: violet, Na: purple, Cs: light green, O: red. Atomic displacement ellipsoids are drawn at a $50 \%$ probability level. 

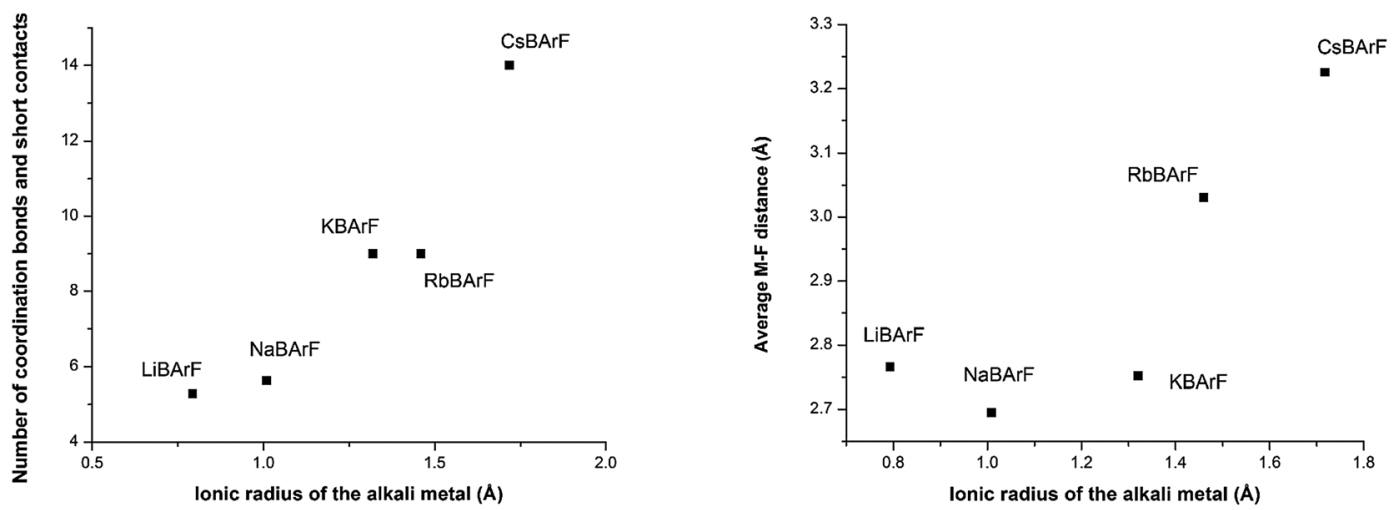

Fig. 5 (Left) The number coordination bonds or/and short contacts around the cationic metal alkali atom compared with the ionic radius of the metal, (right) the average distance between the fluorine atoms and the cationic metal alkali atom compared with the ionic radius of the metal.

metal ${ }^{28}$ is shown on Fig. 5 (left) (this number has been calculated for LiBArF and NaBArF on the basis of the ratio of the octahedral and trigonal bipyramidal structures indicated in the discussion and in the ESI $\dagger$ ). As expected, the trend observed is that the number of coordination bonds and short contacts increases as the ionic radius increases (from $c a$. five in LiBArF to fourteen in CsBArF). Interestingly, the increased number of interactions between the fluorine and the alkali metal cation translates into longer $\mathrm{M}-\mathrm{F}$ distances for cesium, which is the largest cation in the series (Fig. 5 (right)). Interestingly, two reviews by Plenio comprehensively discussed the coordination chemistry of the CF-units to alkali metal centres on the basis of empirical and computational data in the solid-state and in solution. This structural information provided useful data to validate our results. ${ }^{29}$ When considering the shortest M-F distances in the alkali metal BArF salts (see Table 2, entries 5-9), it is observed that all distance values are below the upper threshold for CF-M bond lengths reported by Plenio. ${ }^{29 a}$ On the other hand, the average M-F distances (Fig. 5 (right)) for the Na, $\mathrm{K}$ and Cs derivatives are in agreement with the $\mathrm{CF}-\mathrm{M}$ distance distribution statistics reported in the above mentioned reviews. For the THF-solvates LiBArF-4THF, NaBArF-6THF and CsBArF.7THF, the number of solvate molecules around the metal centres increased as the ionic radius of the metal became larger.

\section{Conclusions}

A new synthetic protocol for the efficient preparation of lithium and potassium BArF has been developed by reaction between $\mathrm{HBArF} \cdot\left(\mathrm{Et}_{2} \mathrm{O}\right)_{2}$ and the corresponding lithium or potassium bis(trimethylsilyl)amide. This new synthesis constitutes an unreported, short, efficient and practical means of synthesising LiBArF and KBArF. The method paves the way for the preparation of new metal BArF derivatives. Moreover, a number of alkali metal and ammonium BArF derivatives have been fully characterised both in solution and in the solid-state by using standard spectroscopic techniques.

Single crystals suitable for X-ray analysis for an array of structurally diverse BArF derivatives were obtained, thus yielding unreported structural details of the structures of KBArF, RbBArF, CsBArF, 6, 7 and 8. The increasing number of coordination bonds and/or short contacts between the alkali metal cation and the surrounding atoms has been rationalised in terms of the size of the alkali metal centre. A study of the structures of alkali metal hydrated and THF-solvates (i.e., LiBArF $\cdot 4 \mathrm{H}_{2} \mathrm{O}$, LiBArF $\cdot 4 \mathrm{THF}$, NaBArF $\cdot 6 \mathrm{THF}$ and CsBArF $\cdot 7 \mathrm{THF}$ ) showed that the interactions between the metal centre and the surrounding atoms depend not only on the size of the alkali metal centre but also on its solvation.

The detailed structural information in the solid-state provided herein regarding an array of alkali metal and ammonium BArF derivatives may well allow a better understanding of the role of BArF derivatives in catalysis, electrochemistry and supramolecular processes and broaden their applicability in future processes of interest.

\section{Acknowledgements}

The authors would like to thank MINECO (CTQ2014-60256-P and Severo Ochoa Excellence Accreditation 2014-2018 SEV2013-0319) and the ICIQ Foundation for financial support. L. C. thanks MINECO for a FPI-SO pre-doctoral fellowship. L. R. and I. M. thank ICIQ for pre-doctoral fellowships. Dr M. V. is grateful for the financial support received from the CELLEX Foundation.

\section{Notes and references}

1 S. H. Strauss, Chem. Rev., 1993, 93, 927-942.

2 I. Krossing and I. Raabe, Angew. Chem., Int. Ed., 2004, 43, 2066-2090.

3 (a) A. G. Massey and A. J. Park, J. Organomet. Chem., 1964, 2, 245-250; (b) H. Kobayashi, T. Sonoda, H. Iwamoto and M. Yoshimura, Chem. Lett., 1981, 579-580; (c) H. Kobayashi, T. Sonoda and H. Iwamoto, Chem. Lett., 1982, 1185-1186; (d) H. Iwamoto, M. Yoshimura, T. Sonoda and H. Kobayashi, Bull. Chem. Soc. Jpn., 1983, 56, 796-801;

(e) H. Nishida, N. Takada, M. Yoshimura, T. Sonoda and H. Kobayashi, Bull. Chem. Soc. Jpn., 1984, 57, 2600-2604; (f) 
K. Fujiki, M. Kashiwagi, H. Miyamoto and A. Sonoda, J. Fluorine Chem., 1992, 57, 307-321; (g) K. Seppelt, Angew. Chem., Int. Ed. Engl., 1993, 32, 1025-1027; (h) J. H. Golden, P. F. Mutolo, E. B. Lobkovsky and F. J. DiSalvo, Inorg. Chem., 1994, 33, 5374-5375; (i) K. Fujiki, J. Ichikawa, H. Kobayashi, A. Sonoda and T. Sonoda, J. Fluorine Chem., 2000, 102, 293-300; (j) E. Bernhardt, G. Henkel, H. Willner, G. Pawelke and H. Burger, Chem.-Eur. J., 2001, 7, 4696-4705.

4 R. F. Jordan, Adv. Organomet. Chem., 1991, 32, 325-387.

5 K. Fujiki, S.-Y. Ikeda, H. Kobayashi, A. Mori, A. Nagira, J. Nie, T. Sonoda and Y. Yagupolskii, Chem. Lett., 2000, 62-63.

6 S. Aldridge, C. Jones, T. Gans-Eichler, A. Stasch, D. L. Kays, N. D. Coombs and D. J. Willock, Angew. Chem., Int. Ed., 2006, 45, 6118-6122.

7 F. Barrière, N. Camire, W. E. Geiger, U. T. Mueller-Westerhoff and R. Sanders, J. Am. Chem. Soc., 2002, 124, 7262-7263.

8 F. Kita, H. Sakata, S. Sinomoto, A. Kawakami, H. Kamizori, T. Sonoda, H. Nagashima, J. Nie, N. V. Pavlenko and Y. L. Yagupolskii, J. Power Sources, 2000, 90, 27-32.

9 A. Boesmann, G. Franciò, E. Janssen, M. Solinas, W. Leitner and P. Wasserscheid, Angew. Chem., Int. Ed., 2001, 40, 26972699.

10 H. Suzuki, H. Naganawa and S. Tachimori, Phys. Chem. Chem. Phys., 2003, 5, 726-733.

11 (a) A. Pfaltz, J. Blankenstein, R. Hilgraf, E. Hormann, S. McIntyre, F. Menges, M. Schonleber, S. P. Smidt, B. Wustenberg and N. Zimmermann, Adv. Synth. Catal., 2003, 345, 33-44; (b) X. Cui and K. Burgess, Chem. Rev., 2005, 105, 3272-3296.

12 (a) I. Mon, D. A. Jose and A. Vidal-Ferran, Chem.-Eur. J., 2013, 19, 2720-2725; (b) A. Vidal-Ferran, I. Mon, A. Bauzá, A. Frontera and L. Rovira, Chem.-Eur. J., 2015, 21, 1141711426; (c) L. Rovira, M. Vaquero and A. Vidal-Ferran, J. Org. Chem., 2015, 80, 10397-10403; (d) H. Fernández-Pérez, I. Mon, A. Frontera and A. Vidal-Ferran, Tetrahedron, 2015, 71, 4490-4494; (e) L. Rovira, H. Fernández-Pérez and A. Vidal-Ferran, Organometallics, 2016, 35, 528-533.

13 (a) Y. Li, B. Ma, Y. He, F. Zhang and Q.-H. Fan, Chem.-Asian J., 2010, 5, 2454-2458; (b) F.-T. Song, G.-H. Ouyang, Y. Li, Y.-M. He and Q.-H. Fan, Eur. J. Org. Chem., 2014, 67136719; (c) Z. Cai, D. Xiao and L. H. Do, J. Am. Chem. Soc., 2015, 137, 15501-15510; (d) X.-C. Zhang, Y.-H. Hu, C.-F. Chen, Q. Fang, L.-Y. Yang, Y.-B. Lu, L.-J. Xie, J. Wu, S. Li and W. Fang, Chem. Sci., 2016, 7, 4594-4599.

14 N. A. Yakelis and R. G. Bergman, Organometallics, 2005, 24, 3579-3581.

15 W. E. Buschmann, J. S. Miller, K. Bowman-James and C. N. Miller, Inorg. Synth., 2002, 33, 83-91.

16 Though seminal pieces of work on alkali metal BArF salts indicate that these compounds can be prepared by cation exchange (see ref. $3 e, 3 h$ and 5), detailed preparation methods and isolated yields are not provided.

17 This statement is made on the basis of the structures checked in the CCDC $\uparrow$ database. A query was made in the CCDC database. The reponse to this query provided all the crystal structures including the $[\mathrm{BArF}]^{-}$sub-structure $(2060$ results) submitted to the CCDC until February 2017 \{CSD version 5.38 [November 2016, update 2 (February 2017)]\}. For CsBArF, only one crystal structure was found (CCDC ref. code FUPBOJ) coordinated to a thioether macrocycle. For RbBArF, two crystal structures were found (CCDC ref. codes FUPBID and OJAMIX) coordinated to a thioether and an aza-macrocycle respectively. For KBArF, six crystal structures were found (CCDC ref. codes: FUPBEZ and FUPCEA (coordinated to a thioether macrocyle), FUPCOK (coordinated to a selenoether macrocyle), OJAMET and OJAMOD (coordinated to an aza-macrocycle), and ZUZVEX (coordinated to a polyether chain)). For LiBArF, eight crystal structures were found (CCDC ref. codes: FUNZUL and FUPCAW (coordinated to a thioether macrocyle), OJAMUJ and OJEBAI (coordinated to an aza-macrocycle), AGOBUV, UQOQOI and YUCGAG (coordinated to a $\mathrm{C}_{60}$ fullerene), and finally a tetrahydrated structure for LiBArF, ref. code: YEMZUL). For NaBArF, 26 crystal structures were found, but only one relevant structure was found (NaBArF $\cdot 2 \mathrm{H}_{2} \mathrm{O}$, CCDC ref. code: RENPIK). None of the ammonium-based BArF salts studied had been indexed by the time of the query.

18 On the contrary, solution and in the solid-state characterisation of alkali metal BArF salts coordinated to a variety of ligands has been recently reported: (a) J. Dyke, W. Levason, M. E. Light, D. Pugh, G. Reid, H. Bhakhoa, P. Ramasami and L. Rhyman, Dalton Trans., 2015, 44, 13853-13866; (b) M. J. D. Champion, W. Levason, D. Pugh and G. Reid, Dalton Trans., 2015, 44, 18748-18759.

19 M. Brookhart, B. Grant and A. F. Volpe Jr, Organometallics, 1992, 11, 3920-3922.

20 The upper temperature limit of the melting point apparatus was $350{ }^{\circ} \mathrm{C}$.

$21(R)$-(1-Phenylethyl)ammonium hydrochloride was synthesised following the reported procedure: K. J. Halloran, A. Comelly, Z. Chen and S. Krishnan, US Pat., WO2011053835A1, 2011.

22 Interestingly LiBArF crystallised also as a tetrahydrate (LiBArF $\left.4 \mathrm{H}_{2} \mathrm{O}\right)$. This specific structure has been reported previously (see ref. $3 h$ ) but the crystallographic study was performed at $173 \mathrm{~K}$. The crystallographic data obtained at $100 \mathrm{~K}$ in the present work for $\mathrm{LiBArF} \cdot 4 \mathrm{H}_{2} \mathrm{O}$ were used for comparison with the LiBArF structures measured under identical conditions. In the case of the $\mathrm{LiBArF} \cdot 4 \mathrm{H}_{2} \mathrm{O}$, the lithium atom is coordinated to four $\mathrm{H}_{2} \mathrm{O}$ molecules in a tetrahedral fashion, and $\mathrm{Li}-\mathrm{F}$ and $\mathrm{B}-\mathrm{Li}$ distances are longer in the tetrahydrate $\mathrm{LiBArF} \cdot 4 \mathrm{H}_{2} \mathrm{O}$ than in LiBArF-A or LiBArF-B (see ESI $\dagger$ ).

23 C.-T. Chang, C.-L. Chen, Y.-H. Liu, S.-M. Peng, P.-T. Chou and S.-T. Liu, Inorg. Chem., 2006, 45, 7590-7592.

24 Pörschke et al. have reported a sixteen-coordinated cesium centre. For example, see: D. Pollak, R. Goddard and K.-R. Pörschke, J. Am. Chem. Soc., 2016, 138, 9444-9451.

25 A. E. H. Wheatley, Chem. Soc. Rev., 2001, 30, 265-273.

26 The measured LiBArF 4 THF sample was a multicomponent crystal formed by a minimum of two crystals (ratio $51: 49$ ). Additionally only a limited number of reflections could be 
collected. Due to these difficulties, the data obtained is less precise.

27 The CsBArF-7THF sample was an inversion twin and presented high disorder in the solvent molecules and $\mathrm{CF}_{3}{ }^{-}$ units. As a result, the bond lengths for a reduced number of $\mathrm{C}-\mathrm{C}$ bonds are less precise.
28 P. F. Lang and B. C. Smith, Dalton Trans., 2010, 39, 77867791.

29 (a) H. Plenio, Chem. Rev., 1997, 97, 3363-3384; (b) H. Plenio, ChemBioChem, 2004, 5, 650-655. 